Bull. Egypt. Soc. Physiol. Sci. 40(2), 86- 102

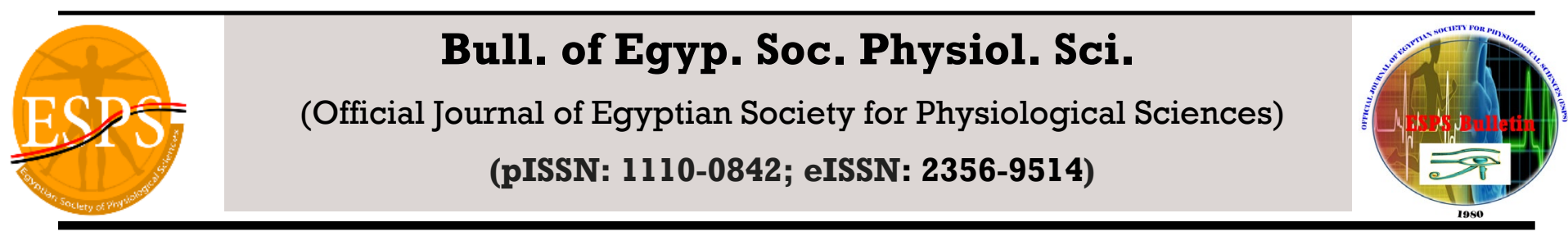

\title{
Functional and Morphological Consequences after Atorvastatin Intake in a Rat Model of Intracerebral Haemorrhage
}

\author{
Noha Badae ${ }^{1}$, Noha Zahran ${ }^{2}$, Azza Baraka ${ }^{3}$, Rasha Elshinety, ${ }^{4}$ Abeer Dief ${ }^{1}$ \\ ${ }^{1}$ Department of Medical Physiology-Alexandria Faculty of Medicine-Egypt, \\ ${ }^{2}$ Department of Histology and Cell biology-Alexandria Faculty of Medicine-Egypt, \\ ${ }^{3}$ Department of Clinical Pharmacology-Alexandria Faculty of Medicine-Egypt, \\ ${ }^{4}$ Department of Anatomy-Alexandria Faculty of Medicine- Egypt.
}

Received:30 Jan 2020

Accepted: 26 April 2020

Available online: 1 July 2020

\section{Keywords}

- Intracerebral haemorrhage

- Statins

- Lipids

- Endothelial cells

- Glial acidic fibrillary protein

- $\mathrm{Ki} 67$

\section{Abstract}

Background: Intracerebral haemorrhage (ICH) is a devastating disease for which there are currently no curative treatment options. Hypolipidemia has been recognized as a possible risk factor for ICH. Several studies have demonstrated that low cholesterol is a risk factor for ICH; others have reported that hypercholesterolemia is protective against ICH. The current study was designed to demonstrate the effect of low LDL levels on vascular endothelial cells integrity and subsequently on the development of ICH in rats. Material and methods: This study was carried on male Wistar albino rats divided into 2 main groups: Group I: normal Sham-operated rats; Group II: ICH rats, were randomly subdivided into 3 subgroups (17 rat each): rats received 2\% gum acacia daily orally; rats received atorvastatin $10 \mathrm{mg} / \mathrm{kg}$ daily alone or with L-arginine $(143 \mathrm{mg} / \mathrm{kg})$ orally for four weeks before induction of ICH and continued for one week after. Behavioral tests were performed 2, 24, and $48 \mathrm{~h}$ after ICH and 7 days later. Serum lipid profile was assessed. Brains were dissected and assessed macroscopically and microscopically for the extent of hematoma, brain water content and endothelin level in the brain homogenate were investigated. Immunoassaying for glial acidic fibrillary protein and Ki67 were performed. Results\& Conclusion: Rats received atorvastatin and L-arginine showed a significant decrease in serum cholesterol and low-density lipoprotein (LDL) concentration. ICH led to marked impairment in motor functions. Atorvastatin and Larginine intake before haemorrhage failed to impose any protection on the motor deficits with corresponding large hematoma size with disruption of nearby tissue. 


\section{INTRODUCTION}

Primary intracerebral haemorrhage (ICH) is a catastrophic condition that is associated with considerable morbidity and mortality with a substantial burden on the patient's family. Notable progression in stroke management leading to a reduction in mortality and disability, however, stroke remains a major cause of death in USA and worldwide (1). Among the risk factors of strokes, hyperlipidaemia remains a subject of ongoing argument (2).

Statins are some of the most commonly prescribed drugs in the fields of cardio-and cerebrovascular disease (3). Despite the ubiquitous usage of statins, it has been noted that some data (from the Heart Protection Study (HPS) and the Stroke Prevention with Aggressive Reductions of Cholesterol Levels (SPARCL)) suggest that the benefits of high-dose statin treatment were partially offset by an increase in haemorrhagic stroke (4). The association of statins and ICH has remained controversial ever since. Subsequent meta-analyses and case control studies reported different results about associated increase in the risk of ICH in patients on statin therapy $(5,6)$. On the contrary, reduced risk of ICH with hypercholesterolemia was reported in several studies (7).

Recently, the potentially harmful effects of nitric oxide (NO) in ICH have emerged from previous studies in neuropathology, which have shown deleterious effects of NO in neurodegeneration (8), traumatic brain injury (9), and stroke (10). The generation of NO in excessive, destructive quantities following a brain injury results largely from activation of the inducible isoform of nitric oxide synthase (iNOS), which occurs in response to inflammatory stimuli. Statins are known to rapidly increase NO bioavailability through multiple mechanisms (11). Statins enhance iNOS expression in vascular smooth muscle cells and eNOS in endothelial cells by cholesterolindependent mechanisms involving Rho and requiring geranylation, leading to prolongation of the half-life of the NOS mRNA (12). Other studies suggested that the possible protective effects against stroke exerted by the use of statin could be attributed to various mechanisms distinct from its lipid-lowering effect (13). Therefore, this study was designed to address the effects of LDL lowing by statin; namely atorvastatin which is a lipid soluble statin that can cross blood brain barrier, on the extent and outcomes of ICH; and whether increasing NO by using a precursor for its synthesis; namely L-arginine, can have a modifying impact on statin effect.

\section{Material \& Methods}

\section{Experimental animals}

All applicable international, national, and/or institutional guidelines for the use of animals were followed and animals were kept in accordance with the guidelines issued by the Ethics Committee of Alexandria faculty of Medicine. Adult male Wistar albino rats weighing 160-200 g were kept under standard laboratory conditions, maintained on a 12-h light-dark cycle, with free access to standard lab chow and water. Rats were divided randomly into 2 main groups. Group I: normal Sham-operated rat $(\mathrm{N}=17)$. Group II: intracerebral haemorrhage group (ICH). ICH group were subdivided into 3 subgroups (17 rat each): rats were given $2 \%$ gum acacia daily orally for four weeks prior to induction of $(\mathrm{ICH})$ and one week after $\mathrm{ICH}$; rats were given atorvastatin in a dose of 
$10 \mathrm{mg} / \mathrm{kg}$ body weight suspended in $2 \%$ gum acacia daily orally for four weeks prior to induction of (ICH) continued for one week after (14). Last group of rats were given atorvastatin as previously in addition to L-Arginine in a dose of $143 \mathrm{mg} / \mathrm{kg}$ daily orally for four weeks prior to induction of ICH and continued for one week after (15).

\section{Induction of ICH:}

Rats were anesthetized by intraperitoneal injection of ketamine/xylazine cocktail (50 mg/kg; 4:1). The animal's head was fixed in the stereotaxic frame. The scalp was cleaned with Betadine solution. Hole was drilled into the skull, a 30-gauge needle was introduced into the striatum $(3 \mathrm{~mm}$ lateral to midline, $0.02 \mathrm{~mm}$ anterior to coronal suture, depth $6 \mathrm{~mm}$ below the surface of the skull) (16). Autologous blood was prepared within 60 secs of infusion (50 $\mu \mathrm{l}$ from retro-orbital vein) and infused into the striatum over 5 minutes. Needle was slowly removed after 10 minutes delay to prevent backflow, the scalp wound then sutured, and the animals were placed in warm cages with free access to food and water (17). Sham operated rats were subjected to the same procedures except for blood infusion.

Body weight of the studied rats was estimated weekly. Serum lipid profile was analyzed at the end of experimental study by enzymatic method. Reagent kits were purchased from BioSystemsS.A. (Costa Brava, 30. 08030 Barcelona, Spain). The analytical performance of these methods was within the manufacturer's specifications (18).

\section{Functional assessment:}

Motor behaviour was evaluated by an observer blinded to the rat groups starting 2 hours after induction of ICH and repeated $24 \mathrm{~h}, 48 \mathrm{~h}$, and 7 days after ICH. All rats subjected to Neurologic deficits score, Beam walking and inverted screen test. Neurologic deficits were scored from $0-4$ as follows; score 0: no neurological deficits, score 1: failure to extend affected forepaw fully (mild focal neurological deficit), score 2: circling to the ipsilateral side (moderate focal neurological deficit); score 3: falling to the ipsilateral side (severe focal neurological deficit) and score 4: no spontaneous movements with depressed level of consciousness (19).

Beam walking is a test used to assess motor coordination and power. Beam balance was conducted as described by Hagemeyeret al (20). Each rat was placed on an elevated horizontal beam $25 \mathrm{~mm}$ in diameter, $60 \mathrm{~cm}$ in length, illuminated at the start side and with a dark little cage at the other end. Each rat trained to walk on the beam for $5 \mathrm{~min}$ daily sessions and the following score system was used: $0=$ Balances with steady posture, $1=$ Grasps the side of the beam, 2 = Hugs the beam and one limb falls from the beam, $3=$ Hugs the beam and two limbs fall from the beam or spins on the beam ( $>60 \mathrm{~s}), 4=$ Attempts to balance on the beam but falls off ( $>$ 40s), 5 = Attempts to balance on the beam but falls off $(>20 \mathrm{~s})$ and $6=$ Falls off with no attempt to balance or hang onto the beam $(<20 \mathrm{~s})$.

Inverted screen test was chosen because it is one of the most feasible ways to assess forelimbs functions. The test was performed with slight modification as reported by Deacon 2014 (21). A $43 \mathrm{~cm}^{2}$ wide wire mesh made up of $12 \mathrm{~mm}$ squares of $1 \mathrm{~mm}$ diameter was used. The screen was held 40-50 $\mathrm{cm}$ above a padded surface. Rats were placed in the center of the wire mesh screen. 
Then, the screen was rotated to an inverted position. The time taken by the rat to fall off the mesh was recorded.

\section{Endothelin essay}

An enzyme-linked immunosorbent assay (ELISA) was used to measure concentrations of Endothelin (ET-1) in brain homogenates, following the manufacturers' instructions. Colour change was measured spectrophotometrically at wavelength $450 \mathrm{~nm}$. Concentrations were normalized against total protein concentration, measured by modified Lowry method. The test kit was supplied by BIOCHEMICA (GesellshaftmbH, Wien Austria) (22).

\section{Brain water content (BWC)}

16 rat brains were utilized for this experiment (4 brains/each group). Twenty-four hours after ICH, rats were killed by pentobarbital overdose. The brains were removed and divided into two hemispheres. The hemispheres were weighed using an electronic analytical balance to obtain the wet weights. Then the brain tissues were dried in an oven at $100^{\circ} \mathrm{C}$ for $24 \mathrm{~h}$ and weighed again. The BWC was calculated according to the following formula: BWC $(\%)=$ (Wet weight-dry weight)/wet weight X100 (23).

\section{Animal sacrifice and histological examination}

One week after ICH, rats were weighed, and then killed by terminal intraperitoneal anesthesia (ketamine $50+$ xylazine $5 \mathrm{mg} / \mathrm{kg}$ ). The brains were excised and both hemispheres were carefully dissected on ice. Both hemispheres were snapfrozen in liquid nitrogen and stored at $-80^{\circ} \mathrm{C}$ for biochemical assays.
Five rats from each group were perfused with $4 \%$ paraformaldehyde in $0.1 \mathrm{~mol} / \mathrm{LPBS}, \mathrm{pH}$ 7.4. Removed brains were kept in $4 \%$ paraformaldehyde (10\% Formol saline) for 24 hours. Fixed brains were cut coronally through the needle entry site (noticeable, on the brain surface), as well as $2 \mathrm{~mm}$ anterior and $2 \mathrm{~mm}$ posterior to that plane. Brain slices were dehydrated and embedded in paraffin. Sections $(5 \mathrm{~mm})$ were cut, and stained with hematoxylin and eosin (16).

For immunohistochemical detection of astrocyte activity and regenerative neurons, GFAP and $\mathrm{Ki}-$ 67 were detected in the deparaffinised sections. The endogenous peroxidase activity was blocked with $10 \%$ hydrogen peroxide for $10 \mathrm{~min}$. To unmask the antigenic sites, sections were placed in $0.01 \mathrm{~mol} / 1$ citrate buffer $(\mathrm{pH}=6)$ in the microwave for $5 \mathrm{~min}$. The slides were washed for $5 \mathrm{~min}$ in PBS at $\mathrm{pH} 7.4$ and then incubated in $1 \%$ BSA dissolved in PBS for $30 \mathrm{~min}$ at $37^{\circ} \mathrm{C}$ to prevent nonspecific background staining. Two drops of ready-to-use primary antibody were applied to sections, and they were incubated for one hour at room temperature. Sections were treated with GFAP (Ab-1, clone GA-5 mouse monoclonal antibody). The slides were rinsed with PBS and a few drops of biotinylated goat-polyvalent secondary antibody were applied for 10 minutes. Note that negative control sections were obtained using PBS without the primary antibody. Anti Ki67 (rabbit polyclonal antibody, sc-15402), Santa Cruz, USA) was diluted 1:200 in PBS. Sections were incubated overnight, followed by washing in PBS and incubation with anti- rabbit IgG, diluted in PBS 1:100 for $1 \mathrm{~h}$ at room temperature. After washing in PBS, sections were incubated with rabbit peroxidase- anti-peroxidase complex (PAP) 
for $1 \mathrm{~h}$ at room temperature. The immunohistochemical signal was developed with diaminobenzidine (DAB) and $\mathrm{H}_{2} \mathrm{O}_{2}$ and sections were counterstained with Mayer's haematoxylin then examined with an Olympus BX 50 photo microscope $(24,25)$.

\section{Morphometric studies of immunostained sections}

Digital images from immunostained sections were obtained using a camera connected to an Olympus BX41 microscope at a magnification of $\times 400$. Random images were selected and analyzed for area percentage and number of astrocytes per high power field. Measurements were done using $\mathrm{NIH}$ Image J (v1.49) (http://rsb.info.nih.gov/ij/).

\section{Statistical Analysis}

Data were presented as mean \pm SEM. All behavioral and histological analyses were performed by experimenter blind to group identity. Most data were analyzed using analysis of variance. However, Mann-Whitney U-test comparisons were used for nonparametric data (i.e., rating scale for Neurological Deficit Scale and beam walking values). A P-value of $<0.05$ was considered statistically significant. Commercially available software package SPSS version 23.0 software (SPSS, Inc., Chicago, IL, USA was used for analysis.

\section{Results}

5 rats developed convulsion and died immediately after stereotaxic surgery, they were replaced by another 5 rats.

\section{Behavioral Changes after ICH}

Medians of neurological deficits score are shown in figure $1 \mathrm{~A}$, the score was significantly impaired 2 and $24 \mathrm{~h}$ after ICH whether treated with Atorvastain and L-Arginine or not compared to control rats. Some improvement starting $48 \mathrm{~h}$ after $\mathrm{ICH}$ in all groups, 7 days after ICH were able to improve the neurological deficit score in all rats with haemorrhage, however, they still have higher deficit scores than controls.

Rats that received an intracerebral infusion of autologous blood showed a significant motor deficit in beam walking compared with controls 24, $48 \mathrm{~h}$ after $\mathrm{ICH}, \mathrm{P}<0.001$. Both Atorvastain or Atorvastain and L-Arginine treated rats exhibited significant poor performance in beam walking compared to control or non-treated rats $(\mathrm{P}<$ 0.001). 7 days after haemorrhage was associated with better beam walking in all groups however, the performance of all rats after haemorrhage is still poor than control (Fig.1 B).

Results of the inverted screen testing are shown in (Fig.1C) compared with sham controls, bloodinfused rats had marked forelimb deficits where time taken by rats to fall off the mesh was significantly decreased from $42.89 \pm 5.48 \mathrm{sec}$ in sham controls to $18.20 \pm 3.48 \mathrm{sec} 2 \mathrm{~h}$ after $\mathrm{ICH}$, the deficit was marked in rats treated with Atorvastain or Atorvastatin and L-Arginine where the duration of grasping was markedly decreased to $5.6 \pm 1.39$ and $4.40 \pm 1.21 \mathrm{sec}$ respectively. 24 or $48 \mathrm{~h}$ after ICH was associated with improvement of grasp strength in all rats, however, the duration was still significantly less than controls $(\mathrm{P}=0.001)$. The inverted screen score still significantly less than shams by day 7 in $\mathrm{ICH}$ rats whether treated by Atorvastatin and L-arginine or not $(\mathrm{P}=0.000)$. However, there was again recovery from this deficit with time. Rats treated with L-Arginine and Atorvastain exhibited better scores in the inverted screen test when compared to rats treated with 
Atorvastatin only especially $48 \mathrm{~h}$ and 7 day after haemorrhage induction.

Lipitor lowered serum cholesterol and LDL levels in treated rats:

Atorvastain intake for 4 weeks either alone or with L-arginine resulted in a significant decrease in cholesterol and LDL concentration of treated rats compared to non-treated ICH rats and controls (P $<0.05)$. Serum TG and HDL showed nonsignificant changes among all groups (Fig. 2 A).

\section{Brain endothelin levels:}

Endothelin level in the brain homogenate showed a significant increase after autologous blood

A

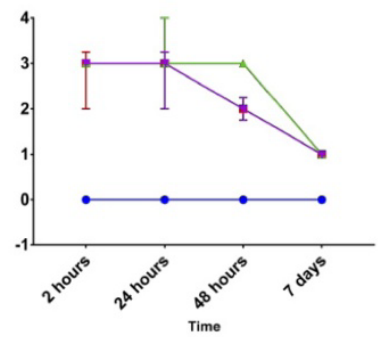

B
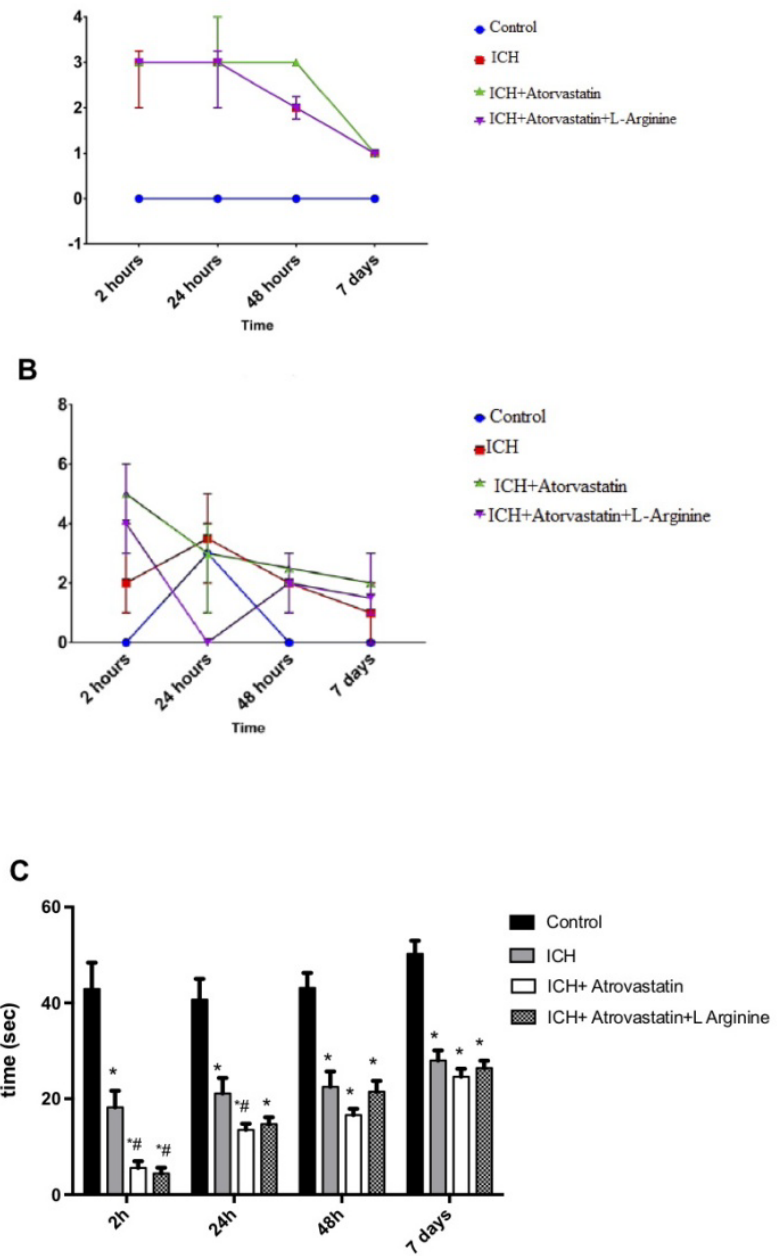

injection compared to sham group $(\mathrm{P}=0.001)$. Rats treated with Atorvastain or with L-Arginine exhibited almost normal endothelin levels as controls which were significantly lower than ICH rats (Fig.2B).

\section{Brain water content (BWC)}

Brain-water content 24 hours after autologous blood injection is shown in (Fig. 2C). Water content was significantly increased in the cerebrum ipsilateral to the hematoma compared to the contralateral side in all animal groups. However, BWC was increased significantly after Atorvastatin intake compared to $\mathrm{ICH}$ rat $(\mathrm{P}=0.01)$. 

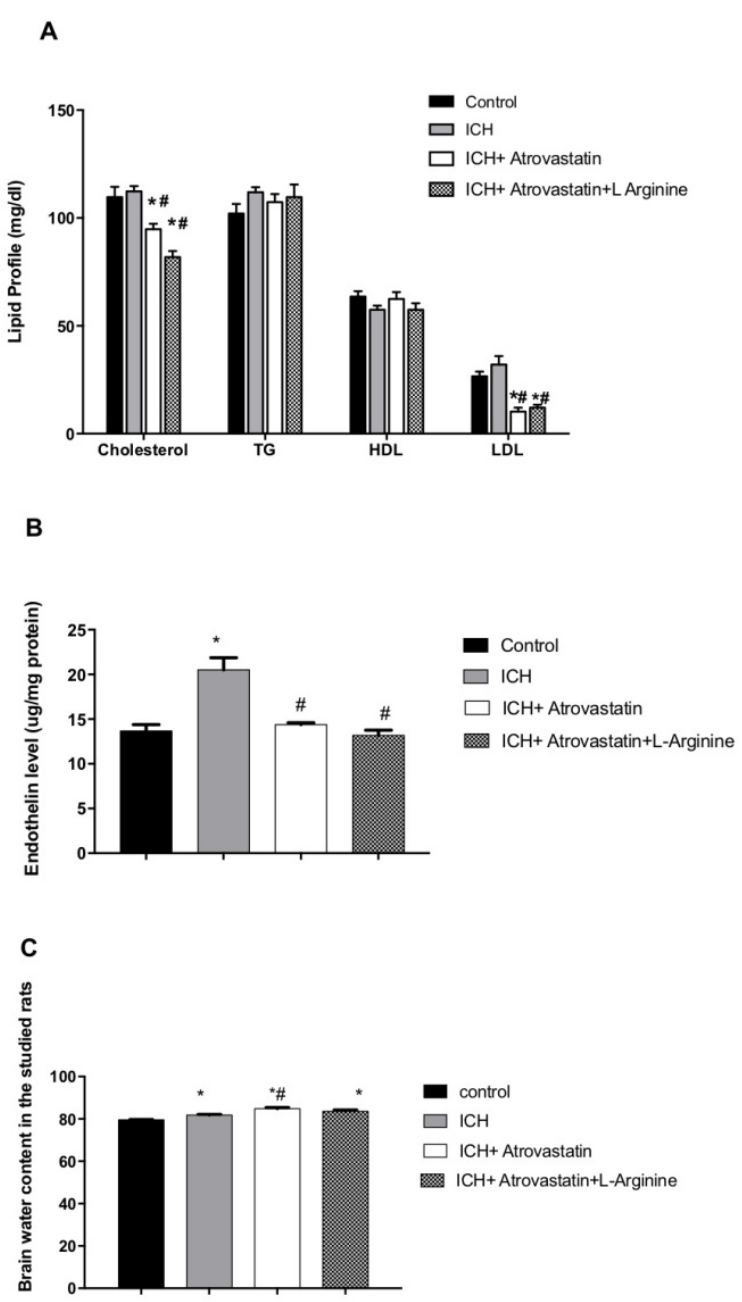

Figure 2: Lipid profile, Endothelin (ET) level and Brain water contents (BWC) in ICH-treated rats and controls. Data presented as means $\pm \mathrm{SEM}$ from $\mathrm{N}=17$ per group. A; lipid profile (mg/dl). B; ET level in the brain homogenate of studied rats ( $\mu \mathrm{g} / \mathrm{mg}$ protein), $\mathrm{C}$; BWC assessed in 4 rats / each group. ANOVA was conducted for all the groups after Atorvastatin and L-Arginine treatment. $* \mathrm{P}<0.05$ vs control \# $\mathrm{P}<0.05$ vs ICH.

Histological and immunohistochemical

\section{findings:}

Schematic diagram illustrates the extent of injury in the ICH models (Fig. 3B) with Atorvastatin (Fig. 3 C) or Atorvastatin and L-Arginine treatment (Fig. 3 D) compared to control rats (Fig. $3 \mathrm{~A})$.

H\&E staining of control rat cerebral hemisphere showed preserved cortical layer architecture with normal acidophilic neuropil (Fig.4 A,B). Rats subjected to autologous blood injection exhibited significantly neuronal loss with disruption of the cortical laminae. Pyramidal neurons appeared shrunken with pyknotic nuclei, neuropil showed reactive gliosis with areas of vasculitis can be noted (Fig 4 D). Thickening of the blood vessel walls were seen with abnormal elongation of the endothelium cell nuclei and inflammatory cells infiltration namely lymphocytes and neutrophils (4 E). Atorvastatin treatment before haemorrhage induction led to massive congestion with increased areas of neuronal loss, more cells with pyknotic nuclei and reactive gliosis and subsequent cellular inflammatory infiltrate. Most of their cells showed vacuolar degeneration. Derangement of cortical layers with pale vacuolated neuropil (Fig.4 
G,H).Rats treated with Atorvastatin and LArginine before haemorrhage induction showed almost the same features seen in rats treated with Atorvastatin only (Fig $4 \mathrm{~J}, \mathrm{~K})$.

Compared to untreated rats with normally appeared hepatocytes and thin walled central vein (Fig. 4 C,F), liver was affected to a great extent by Atorvastatin intake as marked by H\&E staining with thickening of the central vein and wide sinusoidal spaces. Hepatocytes showed vacuolated cytoplasm with dense nuclei and granular cytoplasm (Fig 4 I). L-Arginine treatment showed almost the same features of hepatic reaction as Atorvastatin only (Fig. 4 L).

Immunostaining for GFAP in a cortical and subcortical sample after haemorrhage induction showed numerous GFAP positive glial nuclei with many proliferating processes occur in the haemorrhage area (Fig.5B). Such staining was not noticed in the control astrocytes where they appeared small with few thin processes lying within normal granular and molecular layers (Fig.5A). Atorvastatin intake was associated with decreased GFAP immunoreactivity (Fig.5C) which was also decreased after L-Arginine treatment (Fig.5D). Number of degenerated neurons after Ki 67 immunostaining decreased to some extent after Atorvastatin and L-Arginine treatment (Fig.5 E-H) compared to $\mathrm{ICH}$ rat brain (Fig. 5 F).
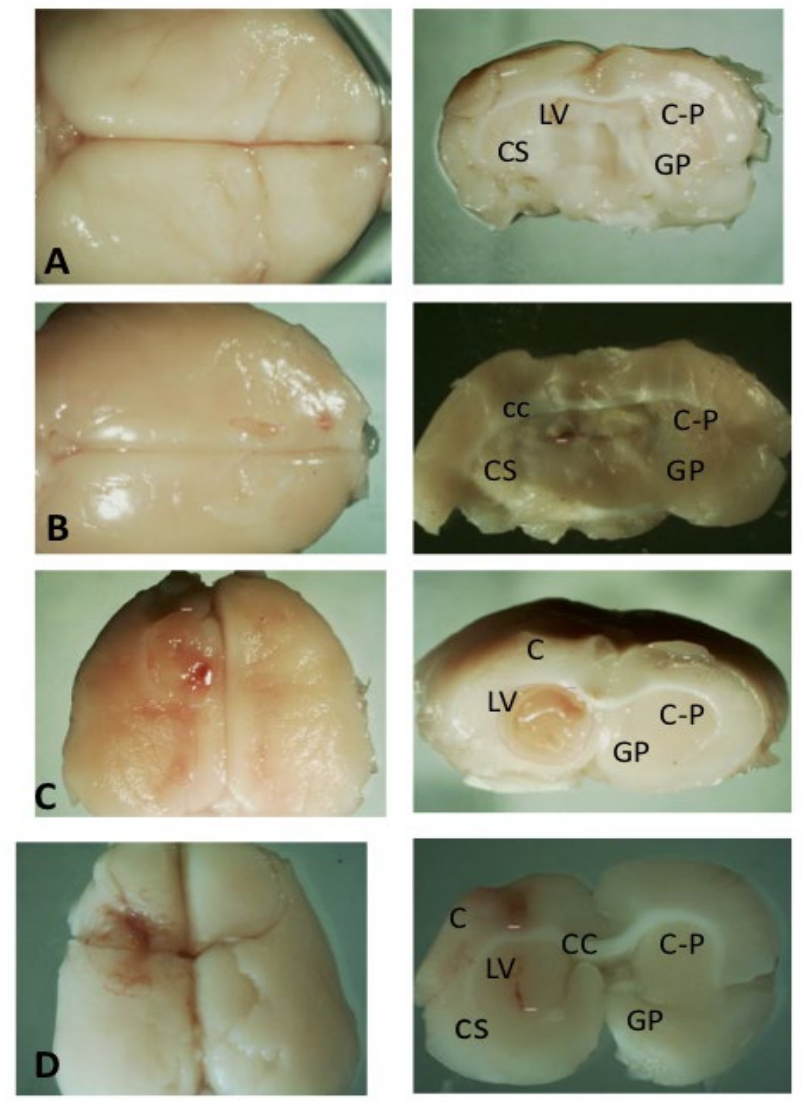

Figure 3: Representative whole brains in control rat with preserved brain architectures (A) and autologous arterial blood injection ICH model illustrates the extent of injury (B) with Atorvastatin (C) and L-Arginine treatment (D). C: Cerebral Cortex; CC: Corpus Callosum; CS: Corpus Striatum; LV: Lateral Ventricle; C-P: Caudate- Putamen; GP: Globus Pallidus 


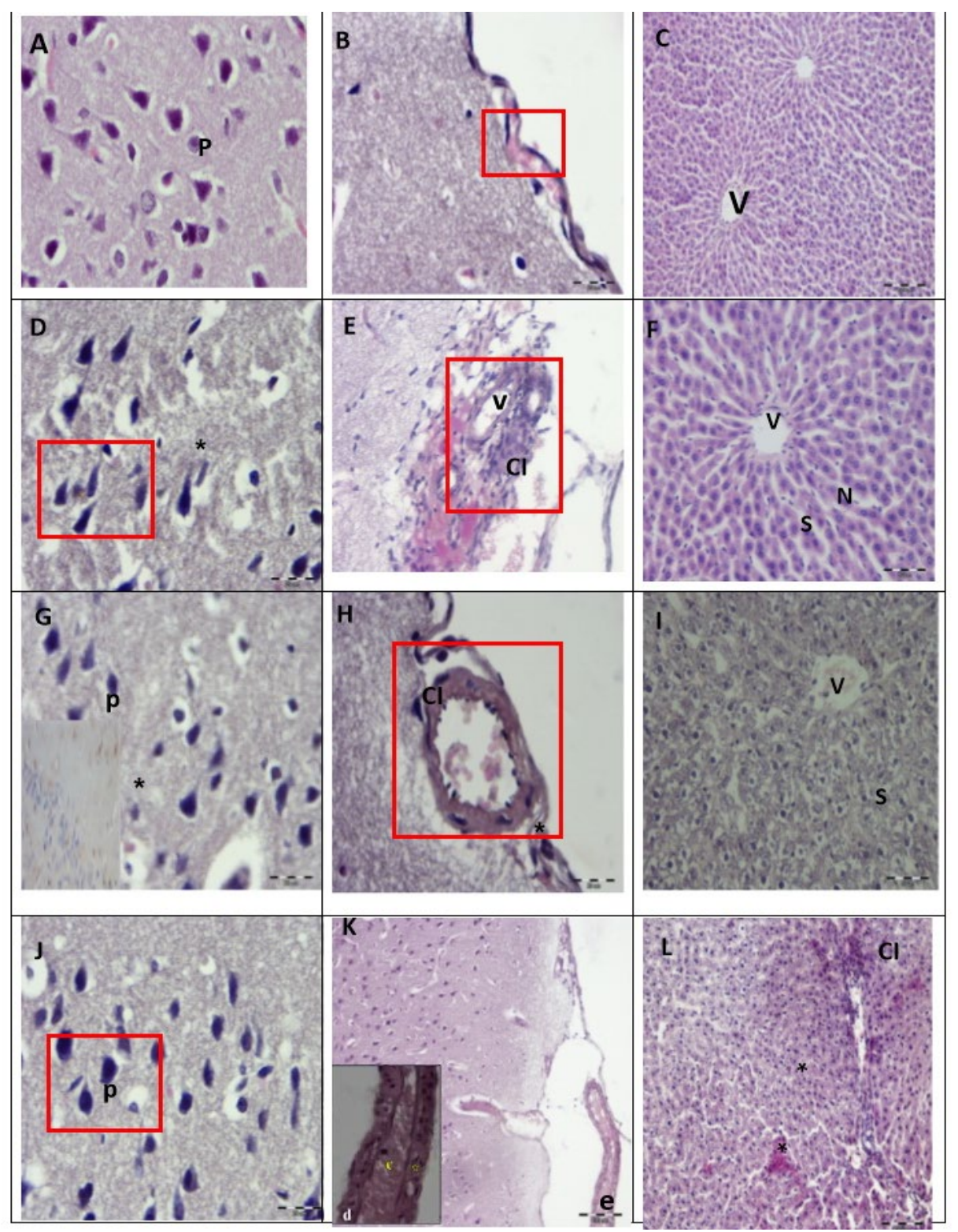

Figure 4: H\&E staining of control rat cerebral hemisphere $(A, B)$ showed preserved cortical layer architecture with normal acidophilic compact neuropil $(\mathrm{N})$ and well defined pyramidal cells $(\mathrm{P})$. Rats subjected to autologous blood injection $(\mathrm{D}, \mathrm{E})$ exhibited significantly neuronal loss with disruption of the cortical laminae. Pyramidal neurons $(\mathrm{P})$ appeared shrunken with pyknotic nuclei, neuropil showed reactive gliosis $\left(^{*}\right)$ with areas of vasculitis and extravasation (red inset) can be noted. Thickening of the blood vessel walls were seen with abnormal elongation of the endothelium cell (e) nuclei and inflammatory cells infiltration (CI) namely lymphocytes and neutrophils. Atorvastatin treatment before haemorrhage induction $(\mathrm{G}, \mathrm{H})$ led to massive congestion with increased areas of neuronal loss, more cells with pyknotic nuclei and reactive gliosis $\left(^{*}\right)$ and subsequent cellular inflammatory infiltrate. Most of their cells showed vacuolar degeneration, Derangement of cortical layers with pale vacuolated neuropil. Thickening of the blood vessel wall (V) was observed with cellular infiltration (CI). Rats treated with Atorvastatin and L-Arginine before haemorrhage induction (J, $\mathrm{K})$ showed almost the same features seen in rats treated with Atorvastatin only. Figure 4 illustrates light photomicrographs of experimental rat liver sections showing: (C,F) hepatocytes of control and ICH non treated rats showed central vesicular nuclei(n), arranged in cords separated by narrow blood sinusoids (S) radiating from thin walled central vein (V) rats treated with Atorvastatin (I) illustrated thick wall central vein (CV) with wide sinusoidal spaces (S) vacuolated hepatocytes $(*)$ and dense nuclei and granular acidophilic cytoplasm $(*)$. Rats treated with Atorvastatin and LArginine (L) showed liver section with pale vesicular nuclei wide sinusoidal spaces (S) and perivascular cellular infiltration $(\mathrm{CI})$. 

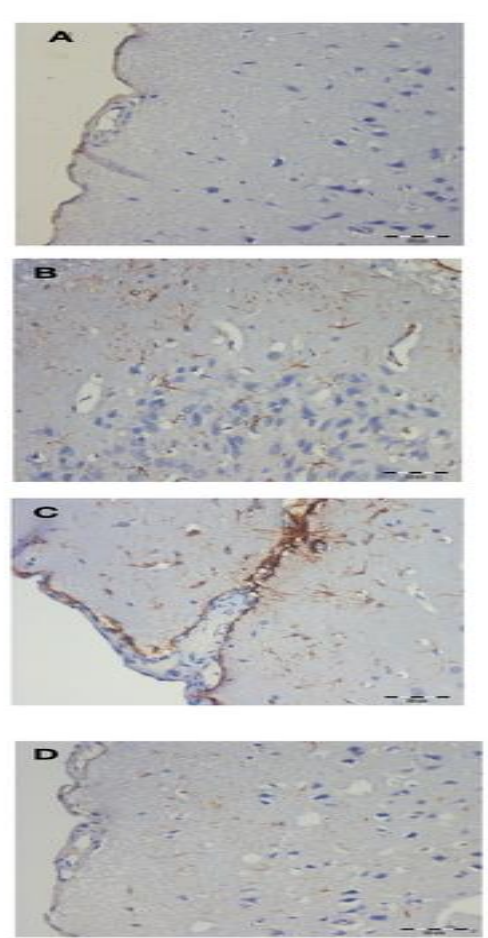
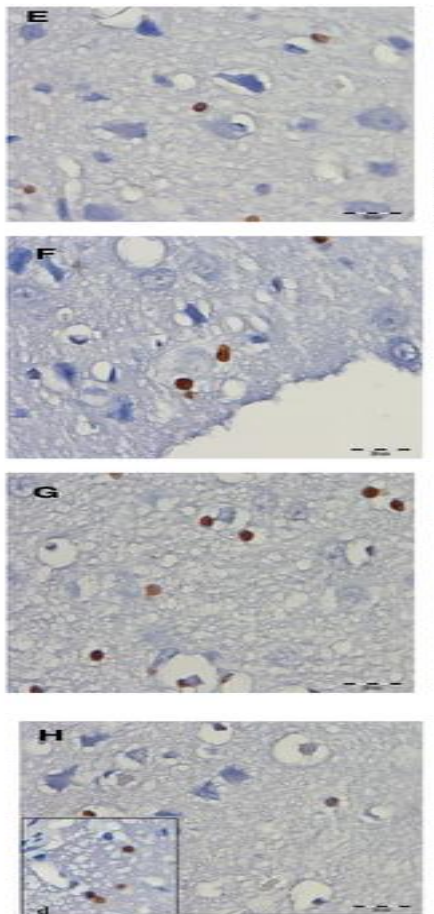
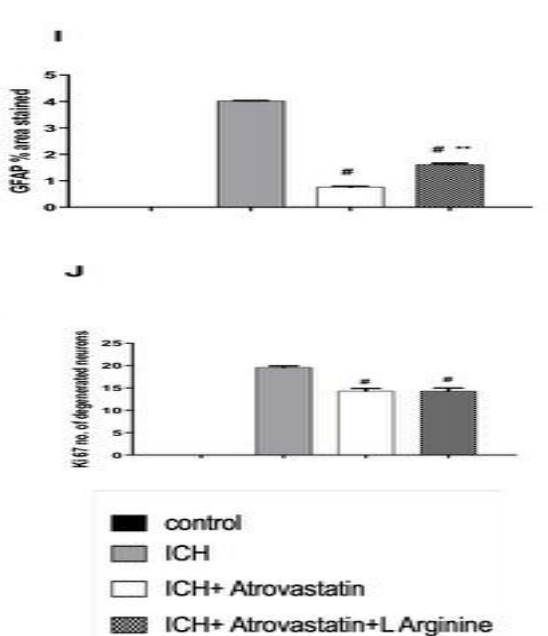

Figure 5: Immunostaining with monoclonal mouse antibodies GFAP of the cerebral hemisphere of studied control rats (A); showed few astrocytic GFAP immunoreactivity is mainly seen within cortical layers and extend around the blood capillaries and beneath the pia matter. Rats injected with autologous blood (B) had a wide spread immunoreactivity for GFAP mainly in the cerebral cortex with glial proliferation. Such immunoreactivity was noticed to a lesser extent after Atorvastatin (C) and L-Arginine treatment (D). Ki 67 positive immunostaining for degenerated neurons and glial cells was seen after hemorrhage induction (F) and decreased slightly after Atorvastatin (G) and L-Arginine (H) intake.

Percent area immunostained with monoclonal mouse antibodies GFAP and no. of degenerated neurons subjected to anti Ki67 (rabbit polyclonal antibody) in the cortical and subcortical tissues from control and ICH rats treated with Atorvastatin and L-Arginine $(\mathrm{I}, \mathrm{J}) . * \mathrm{P}<0.05$ vs control; \# $\mathrm{P}<0.05$ vs ICH; ** $\mathrm{P}<0.05$ vs Atorvastatin.

\section{Discussion}

The benefits of lowering serum cholesterol in stroke patients has not yet been established. Although many studies have been published, most have failed to propose definite mechanism explaining the link between low serum levels of cholesterol and greater risk of intracerebral hemorrhage (26).

The present study revealed a strong association between statin therapy and poor outcomes of ICH. Interestingly rats treated with atorvastatin developed a significant intracerebral hematoma with brain edema after striatal autologous blood injection with impairment of the motor behaviour immediately after ICH. The mechanisms by which statin therapy may increase haemorrhage risk are still questionable. Konishi et al proposed that "decreased serum cholesterol levels contribute to the development of fragile cerebrovascular endothelium, eventually leading to the development of angionecrosis and cerebral haemorrhage (27). Mustonja et al reasoned that "statin therapy could increase the risk of $\mathrm{ICH}$ as low serum cholesterol level may lead to the reduction of vessel resistance to tension, resulting in higher risk of rupture and haemorrhage" (28). Therefore, cholesterol levels are inversely associated with risk of haemorrhagic stroke (2931).

Our study provides additional support for such relation as a positive association has been found between decrease in LDL level and the extent of 
ICH. The association between low total cholesterol and risk of hemorrhage is debatable. Tirschwell et al (32) claimed that the subgroup of patients with the lowest level of total cholesterol had an increased risk of hemorrhagic stroke. There is still considerable uncertainty with regard to the biological explanation for a possible association between low cholesterol and intracerebral haemorrhage. Importantly low cholesterol can contribute to arterial media layer smooth muscle cell necrosis, weakening the vessel wall (33). A further proposed mechanism for an increased risk of ICH with statin therapy is the upregulation of platelet, endothelial nitric oxide synthase expression and platelet-derived nitric oxide release. These effects lead to decreased plateletmediated arterial thrombosis which may be beneficial in preventing ischemic stroke but may be deleterious in ICH (34).

Many researchers, on the other hand, now believe that cholesterol has a critical role in CNS development and synapse support (35). Elevated serum cholesterol is found to have neuroprotective effects by adjusting amino acid uptake and transport, it also counterbalances the effect of free radicals (36).

There is satisfactory agreement among several clinical studies and meta-analysis concerning the role of high cholesterol levels and increased risk of coronary heart disease (37). On the contrary, the relationship between cholesterol and cerebrovascular disease is not clear yet. There is evidence of significantly high mortality from ischemic stroke in patients with high cholesterol levels (38). Lower cholesterol levels on the other hand, have been associated with higher incidence of intracranial hemorrhage (39). Noda et al, demonstrated that "patients with LDL-C $<80$ $\mathrm{mg} / \mathrm{dL}$ or total cholesterol (TC) $<160 \mathrm{mg} / \mathrm{dL}$ had an elevated risk of ICH mortality (40). Therefore, considerable concern about drastic lipid lowering therapy on ICH risk has been raised.

One of the earliest models used for ICH induction is the autologous blood injection, it has the advantages of being clinically relevant and simulate the clinical setting (41). ICH in the current study was associated with severe motor impairment that resolved partially 7days after hemorrhage. Deficits in forelimb placement functions were more pronounced after ICH particularly with atorvastatin or L-arginine treatment. Such impairment was persistent up to 7 days after ICH. Functional recovery after cerebral stroke has been previously observed in previous studies and was attributed to neuronal plasticity rather than neuronal regeneration $(25,42)$.

Research has tended to focus on the protection offered by statin therapy against stroke independent of lipid lowering. Cimino et al, had reported several protective effects of statin therapy as regulation of cerebrovascular tone, possible anti-inflammatory effect and regulation of cerebrovascular tone (43).

Among the adverse effects of ICH is the release of factors from blood clot within the brain such as endothelin (ET). Endothelin is a potent vasoconstrictor with subsequent increase in blood brain barrier permeability and brain edema (44, 45). A previous study on a model of focal cerebral ischaemia revealed an increased level of endogenous ET, $4 \mathrm{~h}$ after ischemia and continued to rise by $24 \mathrm{~h}$ (46). Therefore, significant improvement in cerebral perfusion was demonstrated after the use of ET antagonists which 
may act by antioxidant and anti-inflammatory effects $(47,48)$. Our study confirmed the previous results and demonstrated a significant increase in ET level after $\mathrm{ICH}$, however, rats treated by Atorvastatin only or Atorvastatin and L-Arginine did not exhibit any increase in ET level after ICH. Statin has been found to cause a downregulation of the prepro-endothelin-1 (ET-1) mRNA level in endothelial cells and reduce the ET-1 synthesis (49).

One molecule that has emerged at the center of process of ICH is nitric oxide (NO)"endotheliumderived relaxing factor," $\mathrm{NO}$ is a signaling molecule that has a crucial role in a variety of physiological processes. In the vasculature, NO is important not only as a vasodilator but also for maintaining endothelial function, preventing vascular smooth muscle cell growth, inhibiting platelet aggregation, and blocking leukocyte adhesion. Given that disordered vessel contractility, endothelial dysfunction, inflammation, and free radical injury are all considered to be central to the broader process of $\mathrm{ICH}$, abnormal NO metabolism is uniquely positioned to serve as a central mediator of ICH (50). The possible role of NO in mediating statininduced ICH has been highlighted in the current study where rats treated with L-Arginine (a NO precursor) prior to $\mathrm{ICH}$ had developed motor deficits indistinguishable from non-treated rats as well as non-significant decrease in the hematoma extension. Statins enhance neurotoxic iNOS expression in vascular smooth muscle cells (51). The activation of iNOS induced cell apoptosis in a rat model of cerebral ischemia-reperfusion injury or subarachnoid hemorrhage through induction of caspase 12(52).
Glial fibrillary acidic protein (GFAP) is considered to be a marker for the mature astrocytes in the central nervous system (CNS) (53). Following brain insult as ischemia or hemorrhage, the astrocytes become activated with subsequent release of GFAP to maintain the extracellular environment and provide neuronal protection (54). Yet the definite role of GFAP is not fully understood. Plasma level of GFAP has been recently used as a biomarker for discrimination of intracerebral haemorrhage from acute ischemic stroke (55). The present study shows that treatment with statins decrease GFAP compared to untreated ICH. The present finding was in accordance with Holmberg et al, who proposed that statins decrease astrocyte activation with subsequent decrease in GFAP following CNS injury (56). Regeneration after cerebral insult have been studied previously where the occurrence of reactive astrogliosis was crucial for isolating the necrotic tissue from uninjured surroundings, however, it may impede the regenerative processes.

Another marker that has been found to be elevated in our ICH model and to be slightly decreased by statins is the proliferation marker Ki67. Ki 67 is a nuclear protein normally expressed in G1-, S-, and G2-phases of the cell cycle, where it derived from the resident neural stem cells in various brain regions as subventricular zone and hippocampal subgranular zone $(25,57,58)$. The current study had demonstrated a decreased $\mathrm{Ki} 67$ positive immunostaining of degenerated neurons and glial cells slightly after Atorvastatin intake. Such finding could be explained by antiproliferative effect of statins (59).

A last point to be mentioned is that whilst the effect of statins on the peripheral pool of 
cholesterol is well-established, statins' effects on CNS cholesterol are less clear. The CNS does not rely largely on cholesterol from systemic circulation due to limited metabolic turnover and the brain's inherent capacity to synthesize its own cholesterol (60). As such, reductions in plasma cholesterol concentration following statin treatment are unlikely to cause acute disruption in CNS cholesterol homeostasis (61). Unlike cholesterol in plasma which has a half-life of only a few days (62), brain cholesterol has been associated with a longer half-life. Thus, chronic statin therapy may be required before significant effects on CNS cholesterol are seen, with reductions in CNS cholesterol (63). Because of this, atorvastatin, in our current study, was given for a chronic period of 4 weeks prior to induction of ICH and one week thereafter.

\section{Conclusion}

This work confirmed further evidence demonstrating the relation between low LDL, cholesterol levels and risk of ICH in a rat modal of induced ICH. We underlined the role of damaged endothelium in the development of cerebral hemorrhage. Consequently, the use of intensive lipid lowering medication should be considered cautiously especially in high risk patients. However, the present study has only investigated one of the postulated mechanisms of statin therapy as a risk of hemorrhage. Therefore, further studies assessing other possible mechanistic roles of statins in ICH are needed to be undertaken.

Acknowledgements
The authors extend their thanks to Salma Soliman, graduate Medical student for her contribution to experimental work.

\section{References}

1. Towfighi A, Ovbiagele B, Saver JL. Therapeutic milestone: stroke declines from the second to the third leading organ- and diseasespecific cause of death in the United States. Stroke 2010, 41: 499-503.

2. Amarenco P, Steg PG. The paradox of cholesterol and stroke. Lancet 2007; 370: 1803.

3. Taylor F, Huffman MD, Macedo AF, et al. Statins for the primary prevention of cardiovascular disease. Cochrane Database Syst Rev 2013 ;( 1):CD004816.

4. Gaist D, Goldstein LB, Cea Soriano L, et al. Statins and the risk of intracerebral haemorrhage in patients with previous ischemic stroke or transient ischemic attack. Stroke 2017; 48(12)3245-51.

5. Jhuo SJ, Tsai WC, Lin TH, et al. Statin dose and the risk of intracerebral haemorrhage: a population-based longitudinal study in Taiwan. Acta Cardiol Sin 2016; 32(1):23-30.

6. Brown WV. Safety of statins. Curr Opin Lipidol 2008; 19(6):558-562.

7. Segal AZ, Chiu RI, Eggleston-Sexton PM, et al. Low cholesterol as a risk factor for primary intracerebral hemorrhage: a case-control study. Neuroepidemiology 1999; 18:185-193.

8. Mukherjee P, Cinelli MA, Kang S, et al. Development of nitric oxide synthase inhibitors for neurodegeneration and neuropathic pain. Chem Soc Rev 2014; 43:6814-38. 
9. Cherian L, Hlatky R, Robertson CS. Nitric oxide in traumatic brain injury. Brain Pathol 2004; 14:195-201.

10. Terpolilli NA, Moskowitz MA, Plesnila N. Nitric oxide: Considerations for the treatment of ischemic stroke. J Cereb Blood Flow Metab 2012; 32(7):1332-46.

11. John S, Delles C, Jacobi J,et al. Rapid improvement of nitric oxide bioavailability after lipid-lowering therapy with cerivastatin within two weeks. J Am Coll Cardiol 2001; 37: 1351-8.

12. Rikitake Y, Liao JK. Rho GTPases, statins, and nitric oxide. Circ Res 2005; 97(12):1232-5.

13. Rost N, Rosand J, Woo D. Can hyperlipidemia be protective to the brain? The paradox of lowering lipid levels in cerebrovascular disease. Clinical Lipidology 2010; 5: 295-8.

\section{Yang D, Zhang J, Han Y, James E, Chopp M, Seyfried DM.} Acute statin treatment improves recovery after experimental intracerebral haemorrahge.World J Neurosci 2013; 3(2):69-75.

15. Bilgen I, Öner G, Eren E. Dietary L-Arginine restores Aspirin-induced endothelial dysfunction in rat aorta. Arch Physiol Biochem. 2003; 111(3):232-8.

16. Altumbabic M, Peeling J, Del Bigio MR. Intracerebral hemorrhage in the rat effects of hematoma aspiration. Stroke1998; 29:1917-23.

17. MacLellan CL, Auriat AM, McGie SC, et al. Gauging recovery after hemorrhagic stroke in rats: implications for cytoprotection studies. $\mathrm{J}$ Cereb Blood Flow Metab 2006; 26(8):1031-42.

18. Zhao HL, Sui Y, Guan J, et al. Fat redistribution and adipocyte transformation in uninephrectomized rats. Kidney international 2008; 74(4):467-77.

19. Longa EZ, Weinstein PR, Carlson S. Reversible middle cerebral artery occlusion without craniectomy in rats. Stroke 1989; 20(1):84-91.

20. Hagemeyer N, Boretius S, Ott $\mathbf{C}$, et al. Erythropoietin attenuates neurological and histological consequences of toxic demyelination in mice. Mol Med 2012;18:62835.

21. Deacon RM. Measuring the Strength of Mice. J Vis Exp. 2013;(76).

22. Suzuki K, Matsumoto H, Mijauchi T, et al. Sandwich-enzyme immunoassays for endothelin family peptides. J Cardiovasc Pharmacol 1991; 17 Suppl 7:S420-2.

23. Hua Y, Schallert T, Keep RF, Wu J, Hoff JT, Xi G. Behavioral tests after intracerebral hemorrhage in the rat. Stroke 2002; 33(10):2478-84.

24. Brahmachari S, Fung YK, Pahan K. Induction of glial fibrillary acidic protein expression in astrocytes by nitric oxide. J Neurosci 2006; 26(18):4930-9.

25. Dief AE, Hassaan PS, Hartmut O, et al. Neuronal and glial regeneration after focal cerebral ischemia in rat, an immunohistochemical and electron microscopical study. Alex J of Medicine 2018; 54 (4):699-704.

26. KonishiLauer A, Greenberg SM, Gurol ME. Statins in Intracerebral Hemorrhage. Curr Atheroscler Rep 2015; 17(8):46.

27. Konishi M, Iso H, Komachi $\mathbf{Y}$, et al. Associations of serum total cholesterol, differen $t$ types of stroke, and stenosis distribution of cer 
ebral arteries. The Akita Pathology Study. Stroke 1993; 24(7):954-64.

28. Mustanoja S, Strbian D, Putaala J, et al. Association of prestroke statin use and lipid levels with outcome of intracerebral haemorrhage. Stroke 2013; 44(8):2330-2.

29. Woo D, Kissela BM, Khoury JC, et al. Hypercholesterolemia, HMG-CoA reductase inhibitors, and risk of intracerebral haemorrhage: a case-control study. Stroke 2004; 35(6):1360-4.

30. Phuah CL, Raffeld MR, Ayres AM,et al. Subacute decline in serum lipids precedes the occurrence of primary intracerebral haemorrhage. Neurology 2016; 86(22):203441.

31. Sikora Newsome A, Casciere BC, Jordan JD, et al. The Role of Statin Therapy in Haemorrhagic Stroke. Pharmacotherapy 2015; 35(12):1152-63.

32. Tirschwell DL, Smith NL, Heckbert SR, et al. Association of cholesterol with stroke risk varies in stroke subtypes and patient subgroups. Neurology 2004; 63(10):1868-75.

33. Reiss AB, Wirkowski E. Statins in neurological disorders: mechanisms and therapeutic value. Scientific World Journal 2009; 9:1242-59.

34. koyama S, Ikeda H, Haramaki N, et al. HMG-CoA reductase inhibitor protects against in vivo arterial thrombosis by augmenting platelet-derived nitric oxide release in rats. $\mathrm{J}$ Cardiovasc Pharmacol 2005; 45(4):375-81.

35. Goritz C, Mauch DH, Pfrieger FW. Multiple mechanisms mediate cholesterol-induced synaptogenesis in a CNS neuron. Mol Cell Neurosci 2005; 29(2):190-201.
36. Vatassery GT, Smith WE, Quach HT, et al. In vitro oxidation of vitamin $\mathrm{E}$, vitamin $\mathrm{C}$, thiols and cholesterol in rat brain mitochondria incubated with free radicals. Neurochem Int. 1995; 26(5):527-35.

37. Prospective Studies Collaboration, Lewington S, Whitlock G, Clarke R, et al. Blood cholesterol and vascular mortality by age, sex, and blood pressure: a meta-analysis of individual data from 61 prospective studies with 55,000 vascular deaths. Lancet 2007; 370(9602):1829-39.

38. Wang X, Dong Y, Qi X, et al. Cholesterol levels and risk of haemorrhagic stroke: a systematic review and meta-analysis. Stroke 2013; 44(7):1833-9.

39. Ramírez-Moreno JM, Casado-Naranjo I, Portilla JC, et al. Serum cholesterol LDL and 90-day mortality in patients with intracerebral haemorrhage. Stroke 2009; 40(5):1917-20.

40. Noda H, Iso H, Irie $\mathbf{F}$, et al. Low-density lipoprotein cholesterol concentrations and death due to intraparenchymal haemorrhage: the Ibaraki Prefectural Health Study. Circulation 2009; 119(16):2136-45.

41. Bullock R, Mendelow AD, Teasdale GM, et al. Intracranial haemorrhage induced at arterial pressure in the rat. Part 1: description of technique, ICP changes and neuropathological findings. Neurol Res. 1984; 6(4):184-8.

42. Dief AE, Jirikowski GF, Ragab K, Ibrahim H. Ipsilateral and contralateral cortical apoptosis in rats after unilateral middle cerebral artery occlusion. Anatomy 2008; 2: 39-48.

43. Cimino M, Gelosa P, Gianella A, Nobili E, Tremoli E, Sironi L. Statins: multiple 
mechanisms of action in the ischemic brain. Neuroscientist 2007; 13, 208-213.

44. Matsuo Y, Mihara Si, Ninomiya M,et al. Protective effect of endothelin type A receptor antagonist on brain edema and injury after transient middle cerebral artery occlusion inrats. Stroke 2001; 32(9):2143-8.

45. Lo AC, Chen AY, Hung VK, et al. Endothelin-1 overexpression leads to further water accumulation and brain edema after middle cerebral artery occlusion via aquaporin 4 expression in astrocytic end-feet. J Cereb Blood Flow Metab. 2005; 25(8):998-1011.

46. Barone FC, Globus MY, Price WJ, et al. Endothelin levels increase in rat focal and global ischemia. J Cereb Blood Flow Metab 1994; 14(2):337-42.

47. Gupta YK, Briyal S, Sharma U, et al. Effect of endothelin antagonist (TAK-044) on cerebral ischemic volume, oxidative stress markers and neurobehavioral parameters in the middle cerebral artery occlusion model of stroke in rats. Life Sci. 2005; 77(1):15-27.

48. Mraiche F, Cena J, Das D, et al. Effects of statins on vascular function of endothelin- $1 . \mathrm{Br}$ J Pharmacol. 2005; 144(5):715-26.

49. Förstermann U, Sessa WC. Nitric oxide synthases: Regulation and function. Eur Heart J. 2012; 33(7):829-37, 837a-837d.

50. Iqbal S, Hayman EG, Hong C, et al. Inducible nitric oxide synthase (NOS-2) in subarachnoid hemorrhage: Regulatory mechani smsand therapeutic implications. Brain Circ 2016; 2(1):8-19.

51. Hattori Y, Nakanishi N, Kasai K. Statin enhances cytokine-mediated induction of nitric oxide synthesis in vascular smooth muscle cells. Cardiovasc Res. 2002; 54(3):649-58.

52. Zhao D, Liu Q, Ji Y, et al Correlation between nitric oxide and early brain $\mathrm{i}$ njury after subarachnoid hemorrhage. Int J Neurosci. 2015; 125(7):531-9.

53. Eng LF, Ghirnikar RS, Lee Y L. Glial fibrillary acidic protein: GFAP-thirty-one years (1969-2000). Neurochem Res 2000; 25(910):1439-51.

54. Chen Y, Swanson R A. Astrocytes and brain injury. J Cereb Blood Flow Metab 2003; 23(2):137-49.

55.Katsanos AH, Makris K, Stefani D, et al. Plasma Glial Fibrillary Acidic Protein in the Differential Diagnosis of Intracerebral haemorrhage. Stroke 2017; 48(9):2586-2588.

56. Holmberg E, Zhang SX, Sarmiere PD, et al. Statins decrease chondroitin sulfate proteoglyca $\mathrm{n}$ expression and acute astrocyte activation cent ral nervous system injury. Exp Neurol 2008; 214(1):78-86

57. Scholzen T, Gerdes J. The Ki-67 protein: from the known and the unknown. $\mathrm{J}$ Cell Physiol. 2000; 182(3):311-22.

58. Zhao C, Deng W, Gage FH. Mechanisms and functional implications of adult neurogenesis. Cell 2008; 132(4):645-60.

59. Feldt M, Bjarnadottir O, Kimbung S, et al. Statin-induced anti-proliferative effects via cyclin D1 and p27 in a window-of-opportunity breast cancer trial. J Transl Med 2015; 13:133.

60. Dietschy J M, Turley S D. Cholesterol metabolism in the brain. Curr Opin Lipidol $2001 ; 12: 105-112$.

61. Thelen K M, Rentsch K M, Gutteck U, et al. Brain cholesterol synthesis in mice is affected 
by high dose of simvastatin but not of pravastatin. J Pharmacol Exp Ther 2006; 316:1146-1152.

62. Dietschy J M, Turley S D. Thematic review series: Brain Lipids. Cholesterol metabolism in the central nervous system during early development and in the mature animal. J Lipid Res 2004; 45:1375-1397.

63. Cibičková L. Statins and their influence on brain cholesterol. J Clin Lipidol 2011;5:373379 